\title{
Preventive strategies in depression: gathering evidence for risk factors and potential interventions ${ }^{\dagger}$
}

Michael Berk and Felice Jacka

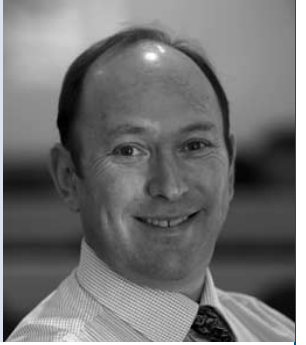

\section{Summary}

This editorial critiques the recent literature concerning both vitamin $\mathrm{D}$ deficiency in major depression and supplementation as a treatment strategy, and contextualises it within a broader approach to the prevention of depression, based on the recent evidence for lifestyle as a risk factor for depression and anxiety.

\section{Declaration of interest}

M.B. has received grant/research support from the $\mathrm{NIH}$ Cooperative Research Centre, Simons Autism Foundation, Cancer Council of Victoria, Stanley Medical Research

Foundation, MBF, the National Health and Medical Research Council (NHMRC), Beyond Blue, Rotary Health, Geelong
Medical Research Foundation, Bristol Myers Squibb, Eli Lilly, Glaxo Smithkline, Organon, Novartis, Mayne Pharma and Servier, has been a speaker for and/or consultant to Astra Zeneca, Bristol Myers Squibb, Eli Lilly, Glaxo SmithKline, Janssen Cilag, Lundbeck, Merck, Pfizer, Sanofi Synthelabo, Servier, Solvay and Wyeth. F.J. has received grant/research support from the Brain and Behaviour Research Institute, NHMRC, Australian Rotary Health, the Geelong Medical Research Foundation, the lan Potter Foundation, Eli Lilly and The University of Melbourne and has been a paid speaker for Sanofi-Synthelabo, Janssen Cilag, Servier, Pfizer, Health Ed, Network Nutrition and Eli Lilly. She is currently supported by an NHMRC Fellowship (\#628912).
Michael Berk (pictured) is Professor of Psychiatry in the School of Medicine, Deakin University, and a Professorial Research Fellow at the Florey Institute of Neuroscience and Mental Health, Orygen Research Centre, and the Department of Psychiatry at the University of Melbourne. Felice Jacka is a Principal Research Fellow in the School of Medicine, Deakin University, and an Honorary Research Fellow in the Department of Psychiatry at the University of Melbourne.

Psychiatry has, to date, lacked a clear evidence base regarding modifiable risk factors for depression and, as a consequence, has been unable to adequately address primary prevention strategies. The discipline has thus focused its attentions on the treatment of established disorders and neglected preventive public health approaches targeting the role of modifiable risk factors. Yet the imperative to increase efforts to prevent depression at a population level is clear.

Although unipolar depression is not officially categorised as a non-communicable disease, the World Health Organization has identified it as the illness accounting for the largest burden of disease in middle- and high-income countries, exceeding that of ischaemic heart disease. However, although lifestyle determinants of other non-communicable diseases have been recognised for decades, surprisingly, the link between environment and lifestyle and risk for psychopathology is a very recent discovery (e.g. Akbaraly et al, ${ }^{1}$ Pasco et $a l^{2}$ Lucas et $a l^{3}$ ).

\section{Vitamin D and depression}

One potentially influential factor is 25-hydroxyvitamin D $(25(\mathrm{OH}) \mathrm{D})$. There is now a substantial body of evidence linking low levels of serum $25(\mathrm{OH}) \mathrm{D}$ to depressive symptoms, paralleling the other health risks from low vitamin D status, such as osteoporosis and cancer. Given that vitamin D is the most prevalent deficiency in developed societies, the report by

'See pp. 360-368, this issue.
Kjærgaard and colleagues in this issue of the Journal is of considerable public health importance. ${ }^{4}$ The study had two aims: first, to examine the relationship between those with low or high serum 25(OH)D levels regarding depressive symptoms; and second, to study, in the subgroup of individuals with low levels of $25(\mathrm{OH}) \mathrm{D}$, the impact of supplementation with $40000 \mathrm{IU}$ vitamin $\mathrm{D}_{3}$ /week for 6 months on depressive symptoms. The study succeeded in demonstrating that individuals with low baseline $25(\mathrm{OH}) \mathrm{D}$ levels had higher depression scores than those with higher $25(\mathrm{OH}) \mathrm{D}$ levels. However, supplementation was of no benefit in addressing depressive symptoms.

Vitamin D has many functions that overlap with the known pathophysiology of depression, which supports the plausibility of a causal role. Vitamin D 25-hydroxylase and $25 \mathrm{D}-1 \alpha$-hydroxylase are expressed in brain areas such as the hypothalamus, cerebellum, substantia nigra and retina. Vitamin $\mathrm{D}$ has a role in sleep and circadian rhythms, and circadian disruption is well documented in depression. ${ }^{5}$ In animal models there is cross-talk between glucocorticoids and vitamin D in the hippocampus; ${ }^{6}$ dysregulated glucocorticoid signalling is also a core component of depression. Vitamin D also influences neuronal growth, cell proliferation in the developing brain and embryogenesis. ${ }^{7}$

\section{Evaluating nutritional treatments}

Well-designed negative studies are important to publish, as they may put to bed hot clinical questions. Since three studies have found a significant effect of high-dose vitamin D supplementation on depressive symptoms, ${ }^{8-10}$ while two others were negative, ${ }^{4,11}$ further studies are needed to establish whether there is a causal relation between vitamin D status and symptoms of depression, what confounding influence physical symptoms might have and, finally, to determine whether correcting vitamin $\mathrm{D}$ status makes any difference. There are substantive differences between treatment and pathophysiology of symptoms and 
threshold clinical disorders, and this is likely to hold true for vitamin $\mathrm{D}$ as well; biological treatments tend to be more useful in threshold disorders and studies will need to examine interventions independently in each group.

Depression therapy is a methodologically treacherous sea to navigate, and Kjærgaard et al's trial illuminates many of the difficulties inherent in the quest. Addressing a single risk factor, which individually accounts for a small percentage of the variance of a complex disorder with many risk factors, allows for a limited scope for change. The use of antidepressants may be a marker of illness acuity; excluding those with prior treatment may leave only a mildly ill group who, based on our understanding of placebo effects in other clinical trials, would bias the sample to have a high placebo response rate and a low likelihood of response to active therapy. That this study examined a non-clinical sample, where treatment effects are less likely, may have reduced the likelihood of success. Last, a cut-off $25(\mathrm{OH}) \mathrm{D}$ level of $55 \mathrm{nmol} / \mathrm{l}$ is quite high, and may not reflect overt clinical deficiency. A signal, if there is any, may lie in individuals with more severe deficiency. As an example, a recent intervention study of folic acid and vitamin $B_{12}$ for the prevention of late-life depression excluded those with severe depression and/or clinically significant vitamin deficiencies on ethical grounds. ${ }^{12}$ Although understandable, this is methodologically problematic, as one would not expect to see an impact of vitamin supplementation on those who were not deficient to start with.

The difficulties in evaluating the impact of vitamin D supplementation on depression apply to all nutritional supplementation in a clinical context. An important consideration when inferring causality in any research examining nutritional status against disease outcomes are the metabolic and behavioural effects of acute illness; in individuals with mental illness, nutrient deficiencies can be caused by the disorder itself. Clearly appetite and self-care are altered in a depressed state. Germane to vitamin $\mathrm{D}$, illness can reduce physical activity and hence sun exposure. ${ }^{13}$ Moreover, stress and acute illness result in significant alterations in nutrient homeostasis, and reduced concentrations of particular nutrients in serum or tissue are observed in those with acute depressive illnesses in the absence of differences in dietary consumption.

In adults with acute illnesses, such as mood disorders, there is a decrease in concentrations of vitamin $\mathrm{E}, \mathrm{C}$ and $\mathrm{A}$, retinol-binding protein, total lipids, pyridoxal-5'-phosphate and albumin, alongside a simultaneous increase in C-reactive protein levels. The inflammatory response is accompanied by an increase in oxidative stress, due to either increased free radical production or inefficient antioxidant systems, which in turn leads to increased lipid peroxidation. Increased oxidative stress is a pathway by which levels of lipids in membranes may be reduced in the presence of depressive illness, amplifying any pre-existing deficiency. This may very well account for the consistent reports of omega-3 fatty acid deficiency in individuals with acute depressive illness. This is supported by the evidence that omega-3 fatty acids are only useful in those with severe depression, where levels are likely to be reduced as a result of illness, and are of no evident protective utility in the general population. Decreases in serum zinc and folate are also seen in patients with major depression, potentially being secondary to the inflammatory response. Indeed, some studies have reported that the resolution of a depressive episode is accompanied by an increase in serum zinc, in the absence of supplementation. What remains to be established is the efficacy of manipulation of the whole diet, as opposed to supplementation of dietary insufficiency. Although there is some, albeit weak, evidence for folate and omega-3 supplementation in depression, the use of other supplements remains unclear. The relative benefits of supplements as opposed to sun exposure are not established.

\section{Lifestyle and depression}

Although many somatic illnesses increase the risk for depression by increasing pain and disability, it is now clear that there are also shared pathophysiological and behavioural risk factors that directly contribute to both somatic and depressive illnesses, including inflammation and oxidative stress. ${ }^{14}$ Lifestyle plays an important role in determining levels of inflammation; consumption of a Mediterranean-style diet, rich in antioxidants, vitamins, minerals and fibre, is associated with reduced systemic inflammation, whereas unhealthy dietary patterns, now common in both low- and middle-income countries and high-income countries, are associated with increased systemic inflammation. The synergistic interactions between the multitudinous components of diet mean that focusing only on individual nutrients may obscure the true associations between diet and mental health. Thus, an examination of the whole diet has greater utility in examining the nutrition-mental health connection. ${ }^{1,15,16}$ Similarly, physical activity is associated with reduced markers of systemic inflammation and may have direct anti-inflammatory effects, while smoking potently increases inflammation and oxidative stress. Another consequence of poor lifestyle practices is obesity, which is a pro-inflammatory state. Obesity and depression share a bidirectional relationship, with obesity potentially contributing to depression via increasing the level of circulating pro-inflammatory cytokines, and depression predisposing to the accumulation of excess adipose tissue. Thus, many of the same pathways whereby poor lifestyle practices contribute to the high-prevalence non-communicable somatic illnesses also appear to influence the risk and progression of common psychiatric illnesses.

While not discounting the possibility of other shared factors, such as genetics, this rapidly developing evidence base suggests that mental and physical illnesses and lifestyle form a triad, with lifestyle as a common denominator. This opens the door to common and integrated treatment approaches based on these shared pathways. It also affords the potential for novel preventive and treatment strategies for these disorders that are the major contributor to the global burden of disease. Given the success of prevention strategies in reducing cardiovascular disease in many countries, this is the next threshold for mental health. Suggested priority actions overlap with those for non-communicable diseases and include government policies to improve the food and built environments and greater funding for prevention programmes.

Michael Berk, MB BCh, MMed(Psych), FF(Psych)SA, FRANZCP, PhD, Deakin

University, School of Medicine and Barwon Health, Geelong, Mental Health Research Institute, Parkville, University of Melbourne, Department of Psychiatry, Parkville, and Orygen Youth Health Research Centre, Parkville, Australia; Felice Jacka, BA, PgDipSci, PhD, Deakin University, School of Medicine and Barwon Health, Geelong, and University of Melbourne, Department of Psychiatry, Parkville, Australia

Correspondence: Professor Michael Berk, Deakin University, P.O. Box 281, Geelong 3220, Australia. Email: mikebe@barwonhealth.org.au

First received 27 Dec 2011, final revision 13 Jun 2012, accepted 5 Sep 2012

\section{References}

1 Akbaraly TN, Brunner EJ, Ferrie JE, Marmot MG, Kivimaki M, Singh-Manoux A. Dietary pattern and depressive symptoms in middle age. Br J Psychiatry 2009; 195: 408-13.

2 Pasco JA, Williams LJ, Jacka FN, Ng F, Henry MJ, Nicholson GC, et al. Tobacco smoking as a risk factor for major depressive disorder: populationbased study. Br J Psychiatry 2008; 193: 322-6. 
3 Lucas M, Mekary R, Pan A, Mirzaei F, O'Reilly EJ, Willett WC, et al Relation between clinical depression risk and physical activity and time spent watching television in older women: a 10-year prospective follow-up study. Am J Epidemiol 2011; 174: 1017-27.

4 Kjærgaard M, Waterloo K, Wang CEA, Almås B, Figenschau Y, Hutchinson MS, et al. Effect of vitamin D supplement on depression scores in people with low levels of serum 25-hydroxyvitamin D: nested case-control study and randomised clinical trial. Br J Psychiatry 2012; 201: 360-8.

5 Turek FW. From circadian rhythms to clock genes in depression. Int Clin Psychopharmacol 2007; 22 (suppl 2): S1-8.

6 Obradovic D, Gronemeyer H, Lutz B, Rein T. Cross-talk of vitamin D and glucocorticoids in hippocampal cells. J Neurochem 2006; 96: 500-9.

7 Eyles D, Burne T, McGrath J. Vitamin D in fetal brain development. Semin Cell Dev Biol 2011; 22: 629-36.

8 Lansdowne AT, Provost SC. Vitamin D3 enhances mood in healthy subjects during winter. Psychopharmacology 1998; 135: 319-23.

9 Jorde R, Sneve M, Figenschau Y, Svartberg J, Waterloo K. Effects of vitamin D supplementation on symptoms of depression in overweight and obese subjects: randomized double blind trial. J Intern Med 2008; 264 599-609.

10 Vieth R, Kimball S, Hu A, Walfish PG. Randomized comparison of the effect of the vitamin D3 adequate intake versus $100 \mathrm{mcg}$ (4000 IU) per day on biochemical responses and the wellbeing of patients. Nutr J 2004; 3: 8 .
11 Sanders KM, Stuart AL, Williamson EJ, Jacka FN, Dodd S, Nicholson G, et al. Annual high-dose vitamin $D_{3}$ and mental well-being: randomised controlled trial. Br J Psychiatry 2011; 198: 357-64.

12 Walker JG, Mackinnon AJ, Batterham P, Jorm AF, Hickie I, McCarthy A, et a Mental health literacy, folic acid and vitamin $B_{12}$, and physical activity for the prevention of depression in older adults: randomised controlled trial. $\mathrm{Br} J$ Psychiatry 2010; 197: 45-54.

13 Bauer M, Glenn T, Alda M, Andreassen OA, Ardau R, Bellivier F, et al. Impact of sunlight on the age of onset of bipolar disorder. Bipolar Disord 2012; 14: $654-63$

14 Pasco JA, Nicholson GC, Williams LJ, Jacka FN, Henry MJ, Kotowicz MA, et al. Association of high-sensitivity C-reactive protein with de novo major depression. Br J Psychiatry 2010; 197: 372-7.

15 Jacka FN, Pasco JA, Mykletun A, Williams $\amalg$, Hodge AM, O'Reilly SL, et al. Association between western and traditional diets and depression and anxiety in women. Am J Psychiatry 2010; 167: 305-11.

16 Sanchez-Villegas A, Delgado-Rodriguez M, Alonso A, Schlatter J, Lahortiga F, Majem LS, et al. Association of the Mediterranean dietary pattern with the incidence of depression: the Seguimiento Universidad de Navarra/University of Navarra follow-up (SUN) cohort. Arch Gen Psychiatry 2009; 66: 1090-8.

\section{psychiatry in the movies}

\section{Vertigo: an overnight success in 54 years}

\section{Peter Byrne}

All of a sudden, vertigo (1958) is the number one film in sight and Sound"s once-in-a-decade poll of 846 critics. Following an inauspicious release, it was a critical slow burner, stealing into 11th place in this poll by 1972. On its initial release, The New Yorker declared: 'Alfred Hitchcock, who produced and directed this thing, has never before indulged in such farfetched nonsense'. Time magazine decided: 'the old master, now a slave to television, has turned out another Hitchcock-and-bull story in which the mystery is not so much who done it as who cares'.

Its plot is initially boy (ex-cop Scottie) meets girl, boy loses girl, boy finds another girl who looks like the first girl, but then goes much darker. Like all great films, it works because interesting things happen to believable characters within the existence of that film. Its themes, sex and death, may have been premature for (at least) its US audiences. It has similarities with the film it displaced at number one, Citizen Kane: flawed protagonist who searches for the unattainable, technical innovation, and a visceral Bernard Hermann score.

There are other reasons that Vertigo almost became a lost classic. Hitchcock switched studios, affecting its promotion and crucially, storage of the original prints. Its 1984 rerelease underwhelmed audiences and critics. In 1997, a remarkable 2-year restoration by Robert Harris and James Katz changed everything. They sourced the original costumes to restore the correctly graded colours from VistaVision to $70 \mathrm{~mm}$ print. Hitchcock had colour coded the film (green for 'go', red for 'stop') to heighten sexual tension. They cleaned and digitalised the sound, instructed by the director's original notes, and even discovered the original music track Hermann recorded in Germany during a US musicians' strike.

And what has all this got to do with psychiatry? Well, we could insist the film be re-titled 'Acrophobia', or opine on the nature of pathological grief. Peter Wollen described the film as a 'visual encyclopaedia of psychopathology'. It has more psychiatry than Hitchcock's earlier psychoanalytic film Spellbound (1945), or his archetypal psychokiller film Psycho (1960). The pathology is not all Scottie's (James Stewart): we the audience are watching him, watching her. Audiences feel his loneliness, driving about a glorious San Francisco, and his vertigo - Hitchcock developed the signature dolly plus zoom technique based on a drunken experience at the Chelsea Arts Ball. To cite a colleague's description of Basil Fawlty: 'there's enough there for an entire conference'. 